\title{
BMJ Open Risk of death among users of Proton Pump Inhibitors: a longitudinal observational cohort study of United States veterans
}

\author{
Yan Xie, ${ }^{1}$ Benjamin Bowe, ${ }^{1}$ Tingting Li, $^{1,2}$ Hong Xian, ${ }^{1,3}$ Yan Yan, ${ }^{1,4}$ Ziyad Al-Aly ${ }^{1,2,5,6}$
}

To cite: Xie Y, Bowe B, Li T, et al. Risk of death among users of Proton Pump Inhibitors: a longitudinal observational cohort study of United States veterans. BMJ Open 2017;7:e015735. doi:10.1136/ bmjopen-2016-015735

- Prepublication history and additional material are available. To view these files, please visit the journal online (http://dx.doi.org/ 10.1136/ bmjopen-2016-015735)

Received 23 December 2016 Revised 20 March 2017 Accepted 22 March 2017

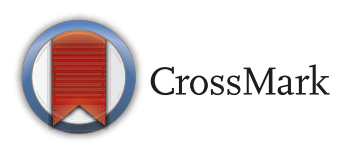

For numbered affiliations see end of article.

Correspondence to

Dr Ziyad Al-Aly;

zalaly@gmail.com

\section{ABSTRACT}

Objective Proton pump inhibitors (PPIs) are widely used, and their use is associated with increased risk of adverse events. However, whether PPI use is associated with excess risk of death is unknown. We aimed to examine the association between PPI use and risk of all-cause mortality.

Design Longitudinal observational cohort study.

Setting US Department of Veterans Affairs.

Participants Primary cohort of new users of PPI or histamine $\mathrm{H} 2$ receptor antagonists ( $\mathrm{H} 2$ blockers) $(\mathrm{n}=349312)$; additional cohorts included PPI versus no PPI $(n=3288092)$ and PPI versus no PPI and no H2 blockers $(\mathrm{n}=2887030)$.

Main outcome measures Risk of death.

Results Over a median follow-up of 5.71 years (IQR 5.11-6.37), PPI use was associated with increased risk of death compared with $\mathrm{H} 2$ blockers use (HR 1.25, $\mathrm{Cl} 1.23$ to 1.28). Risk of death associated with PPI use was higher in analyses adjusted for high-dimensional propensity score (HR 1.16, Cl 1.13 to 1.18), in two-stage residual inclusion estimation (HR 1.21, $\mathrm{Cl} 1.16$ to 1.26 ) and in 1:1 time-dependent propensity score-matched cohort (HR $1.34, \mathrm{Cl} 1.29$ to 1.39). The risk of death was increased when considering PPI use versus no PPI (HR 1.15, Cl 1.14 to 1.15), and PPI use versus no PPI and no $\mathrm{H} 2$ blockers (HR 1.23, Cl 1.22 to 1.24). Risk of death associated with PPI use was increased among participants without gastrointestinal conditions: PPI versus $\mathrm{H} 2$ blockers (HR 1.24, $\mathrm{Cl} 1.21$ to 1.27), PPI use versus no PPI (HR 1.19, $\mathrm{Cl} 1.18$ to 1.20 ) and $\mathrm{PPI}$ use versus no $\mathrm{PPI}$ and no $\mathrm{H} 2$ blockers (HR 1.22, $\mathrm{Cl} 1.21$ to 1.23). Among new PPI users, there was a graded association between the duration of exposure and the risk of death.

Conclusions The results suggest excess risk of death among PPI users; risk is also increased among those without gastrointestinal conditions and with prolonged duration of use. Limiting PPI use and duration to instances where it is medically indicated may be warranted.

\section{INTRODUCTION}

Proton pump inhibitors (PPI) are widely prescribed and are also available for sale over the counter without prescription in several countries. ${ }^{1}{ }^{2}$ Several observational studies suggest that PPI use is associated with

\section{Strengths and limitations of this study}

- National large-scale data from a network of integrated health systems.

- Employed a new user design and developed a number of analytical approaches where we consistently found a significant association between PPI exposure and risk of death

- Cohort included mostly older white male US veterans, which may limit the generalisability.

Did not include information on the cause of death.

increased risk of a number of adverse health outcomes. ${ }^{1}$ A number of studies have shown that PPI use is associated with significant risk of acute interstitial nephritis. ${ }^{3-5}$ Recent studies established an association between exposure to PPI and risk of chronic kidney disease (CKD), kidney disease progression and end-stage renal disease. ${ }^{267}$ Results from a large prospective observational German cohort suggest that patients receiving PPI had a higher risk of incident dementia. ${ }^{8}$ Several reports highlighted a rare but potentially fatal risk of hypomagnesemia among users of PPI. ${ }^{9-11}$ PPI use has been associated with increased risk of both incident and recurrent Clostridium difficile infections. ${ }^{12}$ Several observational analyses have shown that PPI use was also associated with increased risk of osteoporotic fractures, including hip and spine fractures. ${ }^{13}{ }^{14}$ Less convincing-and to some extent inconsistent-evidence suggests a relationship between PPI use and risks of community-acquired pneumonia and cardiovascular events. ${ }^{15-17}$ Emerging-and far from conclusive-in vitro evidence suggests that PPI results in inhibition of lysosomal acidification and impairment of proteostasis, leading to increased oxidative stress, endothelial dysfunction, telomere shortening and accelerated senescence in human endothelial cells. ${ }^{18}$ The experimental work provides a 
putative mechanistic link to explain some of the adverse events associated with PPI use. ${ }^{18}$

The adverse outcomes associated with PPI use are serious, and each is independently associated with higher risk of mortality. Evidence from several small cohort studies of older adults who were recently discharged from the hospital or institutionalised in long-term care facilities suggests inconsistently that PPI use may be associated with increased risk of 1 year mortality. ${ }^{19-22}$ Whether PPI use is associated with excess risk of death is not known and has not been examined in large epidemiological studies spanning a sufficiently long duration of follow-up. We hypothesised that owing to the consistently observed associations between PPI use and risk of adverse health outcomes, PPI use is associated with excess risk of death, and that the risk of death would be more pronounced with increased duration of use. We therefore used the Department of Veterans Affairs national databases to build a longitudinal cohort of incident users of acid suppression therapy, including PPI and histamine $\mathrm{H} 2$ receptor antagonists (H2 blockers), to examine the association between PPI use and risk of all-cause mortality and to determine whether risk of death is increased with prolonged duration of use.

\section{METHODS \\ Cohort participants \\ Primary cohort}

Using administrative data from the US Department of Veterans Affairs, we identified patients who received an outpatient H2 blockers or PPI prescription between 1 October 2006 and 30 September 2008 ( $n=1762908)$. In order to select new users of acid suppression therapy (incident user design), we excluded 1356948 patients who received any outpatient H2 blockers or PPI prescriptions between 1 October 1998 and 30 September 2006. To account for patients' kidney function, only patients with at least one outpatient serum creatinine value before the first acid suppression therapy prescription were selected in the cohort, yielding an analytic cohort of 349312 patients. Patients whose first acid suppression therapy was PPI ( $n=275977)$ were considered to be in the PPI group during follow-up. Patients who received H2 blockers as their first acid suppression therapy $(\mathrm{n}=73335)$ served as the reference group before they received any PPI prescription (see online supplementary figure 1). Within the reference group, those who received a PPI prescription later $(n=33136)$ were considered to be in the PPI group from the date of their first PPI prescription until the end of follow-up. ${ }^{23}$ Time zero (T0) for primary cohort was defined as the first acid suppression therapy prescription date.

\section{Secondary cohorts}

We additionally built two secondary cohorts to examine the association of PPI use and risk of death in (a) PPI versus no PPI users and (b) PPI versus non-users of acid suppression therapy. Patients with no PPI prescription between 1 October 1998 and 30 September 2006, and with at least one outpatient eGFR value before 1 October 2006, were selected to evaluate the risk of death associated with PPI use versus no PPI use ( $n=3$ 288 092) (see online supplementary figure 2a). Patients with no PPI prescription between 1 October 1998 and 30 September 2006, with no $\mathrm{H} 2$ blockers before the first PPI prescription and at least one outpatient eGFR value before 1 October 2006, were selected to evaluate the risk of death associated with PPI use versus no acid suppression therapy ( $\mathrm{n}=2887030$ ) (see online supplementary figure $2 \mathrm{~b}$ ). T0 for secondary cohorts was defined as 1 October 2006.

Patients in both primary and secondary cohorts were followed until 30 September 2013 or death. The study was approved by the Institutional Review Board of the VA Saint Louis Health Care System, Saint Louis, Missouri.

\section{DATA SOURCES}

We used the Department of Veterans Affairs databases, including inpatient and outpatient medical SAS data sets (that include utilisation of data related to all inpatient and outpatient encounters within the VA system), to ascertain detailed patient demographic characteristics and comorbidity information based on inpatient and outpatient encounters. ${ }^{24}$ The VA Managerial Cost Accounting System Laboratory Results (a comprehensive database that includes VA-wide results for selected laboratory tests obtained in the clinical setting) provided information on outpatient and inpatient laboratory results. The VA Corporate Data Warehouse Production Outpatient Pharmacy domain provided information on outpatient prescriptions. The VA Vital Status and Beneficiary Identification Records Locator Subsystem files provided demographic characteristics and death.

\section{Primary predictor variable}

PPI use was the primary predictor. Once cohort participants received PPI prescription, they were considered with the effect of PPI until the end of follow-up. Medications that contain esomeprazole, lansoprazole, omeprazole, pantoprazole or rabeprazole were counted as PPI. Medications including ranitidine, cimetidine and famotidine were counted as H2 blockers.

\section{Outcome}

The primary outcome in survival analyses was time to death. Death information is routinely collected by the Veterans Benefit Administration for all United States Veterans.

\section{Covariates}

Covariates included age, race, gender, eGFR, number of outpatient serum creatinine measurements, number of hospitalisations, diabetes mellitus, hypertension, cardiovascular disease, peripheral artery disease, cerebrovascular disease, chronic lung disease, cancer, hepatitis C, HIV, dementia and diseases associated with acid suppression 
therapy use such as gastro-oesophageal reflux disease (GERD), upper gastrointestinal (GI) tract bleeding, ulcer disease, Helicobacter pylori infection, Barrett's oesophagus, achalasia, stricture and oesophageal adenocarcinoma. ${ }^{25-28}$ eGFR was calculated using the abbreviated four-variable CKD epidemiology collaboration equation based on age, sex, race and outpatient serum creatinine. ${ }^{29}$ Race/ ethnicity was categorised as white, black or other (Latino, Asian, Native American or other racial/ethnic minority groups). Comorbidities except for hepatitis $\mathrm{C}$ and HIV were assigned on the basis of relevant ICD-9-CM (the International Classification of Diseases, Ninth Revision, Clinical Modification) diagnostic and procedure codes and Current Procedural Terminology (CPT) codes in the VA Medical SAS data sets. ${ }^{20-33}$ Hepatitis C and HIV were assigned based on laboratory results.

Baseline covariates were ascertained from 1 October 1998 till T0. All covariates except for age, race and gender covariates values were treated as time-varying covariates where they were additionally assessed until the date of the first PPI prescription in those patients who did not have PPI prescription at T0. Any comorbidity occurring during the assessment period was considered present during the remaining follow-up. eGFR was the outpatient eGFR value within and most proximate to the end of the assessment period. Number of outpatient serum creatinine measurements and number of hospitalisations were accumulated during the assessment period.

\section{Statistical analysis}

Means, SD and t-tests are presented for normally distributed continuous variables; medians, interquartile ranges and Wilcoxon-Mann-Whitney tests are presented for non-normally distributed continuous variables; and counts, percentages and $\chi^{2}$ tests are presented for categorical variables. Incident rates per 100 person-years were computed for death, and CIs were estimated based on the normal distribution. The Simon and Makuch method for survival curves was used for time-dependent covariates. ${ }^{34}$

Cox regression models with time-dependent covariates were used in the assessment of the association between PPI exposure and risk of death where patients could switch from H2 blockers to PPI in the models. In order to account for potential delayed effect of PPI, patients were considered to have the effect of PPI from the first PPI prescription till the end of follow-up. In addition, time-dependent Cox models were conducted in subgroups where patients had no GI conditions and where patients had no GI conditions except for GERD and in the secondary cohorts.

Because exposure in this observational cohort is time dependent, we undertook 1:1 propensity score matching for the primary cohort where time-dependent propensity scores were calculated based on time-dependent Cox regression with all covariates ${ }^{35}$ (details are provided in online supplementary methods). After matching, all covariates except for age had an absolute standardised difference of less than 0.1, which indicated that all covariates except age were well balanced. Age had a standardised difference equal to 0.13 . Doubly robust estimation was applied after matching, where all covariates were additionally controlled for in the model to obtain an unbiased effect estimator. ${ }^{36}$

In order to optimise control of confounding, we additionally built high-dimensional propensity score-adjusted survival models following the multistep algorithm described by Schneeweiss et $a \hat{l}^{\beta 7}$ (details are provided in online supplementary methods). We also applied a two-stage residual inclusion estimation based on instrumental variable approach (see online supplementary methods) ${ }^{38}$

In addition, we evaluated the association between duration of PPI prescription and risk of death among new users of PPI. Duration was defined in cumulative days of use and categorised as $\leq 30,31-90,91-180,181-360$ and $361-720$, where $\leq 30$ days was considered as the reference group. To avoid immortal time bias (by definition, cohort participants must be alive to receive prescription hence introducing a bias commonly referred to as immortal time bias), time of cohort entry was defined as the date of last PPI prescription plus days' supply. ${ }^{39} 40$ In order to ensure sufficient length of follow-up time following T0, we excluded cohort participants with cumulative duration of exposure exceeding 720 days (because of limited overall cohort timeline, and because T0 starts at the end of last prescription, those with long exposure will necessarily have limited follow-up time). In regression analyses, a 95\% CI of an HR that does not include unity was considered statistically significant. All analyses were performed using SAS Enterprise Guide version 7.1.

\section{Sensitivity analyses}

In order to further evaluate the consistency and robustness of study findings, we examined the observed associations in a less contemporary cohort (dating back to an era where PPI prescription and use were far less frequent) of patients without acid suppression therapy prescriptions between 1 October 1998 and 30 September 2000 (washout period) and with acid suppression therapy prescription between 1 October 2000 and 30 September 2002 and at least one outpatient serum creatinine value before that. Patients in this cohort were followed till 30 September 2007 or death. To examine the impact of potential residual confounding on study results, we conducted additional sensitivity analyses as described by Schneeweiss ${ }^{41}$ : (a) we used the rule-out approach to identify the strength of the residual confounding that could fully explain the association observed in primary analyses, and (b) we applied an external adjustment approach using external information (prevalence and risk estimates from published literature) to evaluate potential net confounding bias due to unmeasured confounders. ${ }^{2}{ }^{41-44}$ Methods are described elegantly by Schneeweiss. ${ }^{41}$ In addition, to remove death events that were less likely to be related to PPI exposure, we excluded cohort participants 
who died within 90 days after the first PPI or H2 blocker prescription.

We conducted analyses based on a three-level classification of exposure, where patient's status at time t could be current use (using PPI or finished last PPI prescription within 90 days before $t$ ), past use (used PPI after $T_{0}$ but finished more than 90 days before t) and never use. We conducted additional sensitivity analyses, which included haemoglobin as a covariate in cohort participants with available data. We also undertook analyses that stratified the cohort based on cardiovascular disease, history of pneumonia, CKD (eGFR $<60$ and $\geq 60 \mathrm{~mL} / \mathrm{min} / 1.73 \mathrm{~m}^{2}$ ) or age ( $<65$ and $\geq 65$ years old) at $\mathrm{T}_{0}$. Finally, and in order to ascertain the specificity of the findings, we examined the association between PPI exposure and the risk of a motor vehicle accident as a tracer outcome where a priori knowledge suggests an association is not likely to exist.

\section{Patient involvement}

No patients were involved in developing the hypothesis, the specific aims or the research questions, nor were they involved in developing plans for design or implementation of the study. No patients were involved in the interpretation of study results or write up of the manuscript. There are no plans to disseminate the results of the research to study participants or the relevant patient community.

\section{RESULTS}

The demographic and health characteristics of the overall primary cohort of new users of acid suppression therapy $(\mathrm{n}=349312)$, by type of acid suppressant drug at time of cohort entry (H2 blockers $n=73335$; PPI $n=275977)$, and those who were ever exposed to PPI $(n=309113)$ are provided in table 1 . There were significant baseline differences in that cohort participants who were treated with PPI were older and were more likely to have comorbid conditions, including diabetes, hypertension, cardiovascular disease and hyperlipidaemia. Cohort participants treated with PPI were also more likely to have upper GI tract bleeding, ulcer disease, H. pylori infection, Barrett's oesophagus, achalasia, stricture and oesophageal adenocarcinoma (table 1). Survival curves for PPI and H2 blockers are presented in figure 1 .

\section{Association between PPI use and risk of death}

Among new users of acid suppression therapy ( $n=349312)$, and over a median follow-up of 5.71 years (IQR 5.116.37), where exposure was treated as a time-dependent covariate, PPI use was associated with increased risk of death compared with H2 blockers use (HR 1.25, CI 1.23 to 1.28) (table 2). Among new users of acid suppression therapy ( $\mathrm{n}=349312)$, in high-dimensional propensity score-adjusted models, new PPI users had increased risk of death compared with new users of $\mathrm{H} 2$ blockers (HR 1.16 , CI 1.13 to 1.18 ); based on two-stage residual inclusion estimation, risk of death was higher in new PPI users when compared with new users of H2 blockers (HR 1.21,
CI 1.16 to 1.26$)$. In a 1:1 time-dependent propensity scorematched cohort of new users of PPI and H2 blockers $(n=146670)$, PPI users had significantly increased risk of death (HR 1.34, CI 1.29 to 1.39).

We examined the relationship of PPI and risk of death in secondary cohorts (as described in the Methods section) where we considered risk associated with PPI use versus no known exposure to PPI (no PPI use $\pm \mathrm{H} 2$ blockers use) ( $n=3288092)$; the results suggest that PPI use was associated with increased risk of death (HR 1.15, CI 1.14 to 1.15) (table 2). Assessment of risk of death associated with PPI use versus no known exposure to any acid suppression therapy (no PPI use and no H2 blockers use) $(n=2887070)$ suggests increased risk of death with PPI use (HR 1.23, CI 1.22 to 1.24).

\section{Association between PPI use and risk of death in those without GI conditions}

We then analysed the association between PPI use and risk of death in cohort where we excluded participants with documented medical conditions generally considered as indications for treatment with PPI, including GERD, upper GI tract bleeding, ulcer disease, H. pylori infection, Barrett's oesophagus, achalasia, stricture and oesophageal adenocarcinoma. The intent of this analysis was to examine the putative association of PPI use and risk of death in a lower risk cohort. Examination of risk of death associated with use of acid suppression therapy (PPI vs H2 blockers) suggests that risk of death was increased with PPI use (HR 1.24, CI 1.21 to 1.27) (table 2). Examination of the risk of death associated with PPI use versus no known exposure to PPI (no PPI use $\pm \mathrm{H} 2$ blockers use) suggests a higher risk of death associated with PPI use (HR 1.19, CI 1.18 to 1.20). Results were consistent where we examined risk of death associated with PPI use versus no known exposure to any acid suppression therapy (no PPI use and no H2 blockers use) (HR 1.22, CI 1.21 to 1.23). Risk of death associated with PPI use in cohort participants without GI conditions but included participants with GERD yielded consistent results (PPI vs H2 blockers (HR 1.24, CI 1.21 to 1.27); PPI vs no PPI (HR 1.14, CI 1.13 to 1.14 ); PPI vs no PPI and no H2 blockers (HR 1.22, CI 1.21 to 1.22 )) (table 2).

\section{Duration of exposure and excess risk of death}

We examined the association between duration of PPI exposure and risk of death among new users of PPI $(n=166098)$. Compared with those exposed for $\leq 30$ days, there was a graded association between duration of exposure and risk of death among those exposed for 31-90, 91-180, 181-360 and 361-720 days (table 3, figure 2).

\section{SENSITIVITY ANALYSES}

We tested the robustness of study results in sensitivity analyses where we built a less contemporary cohort as described in the Methods section; demographic and health characteristics of this cohort are provided in online supplementary table 1 . Where exposure was treated as 


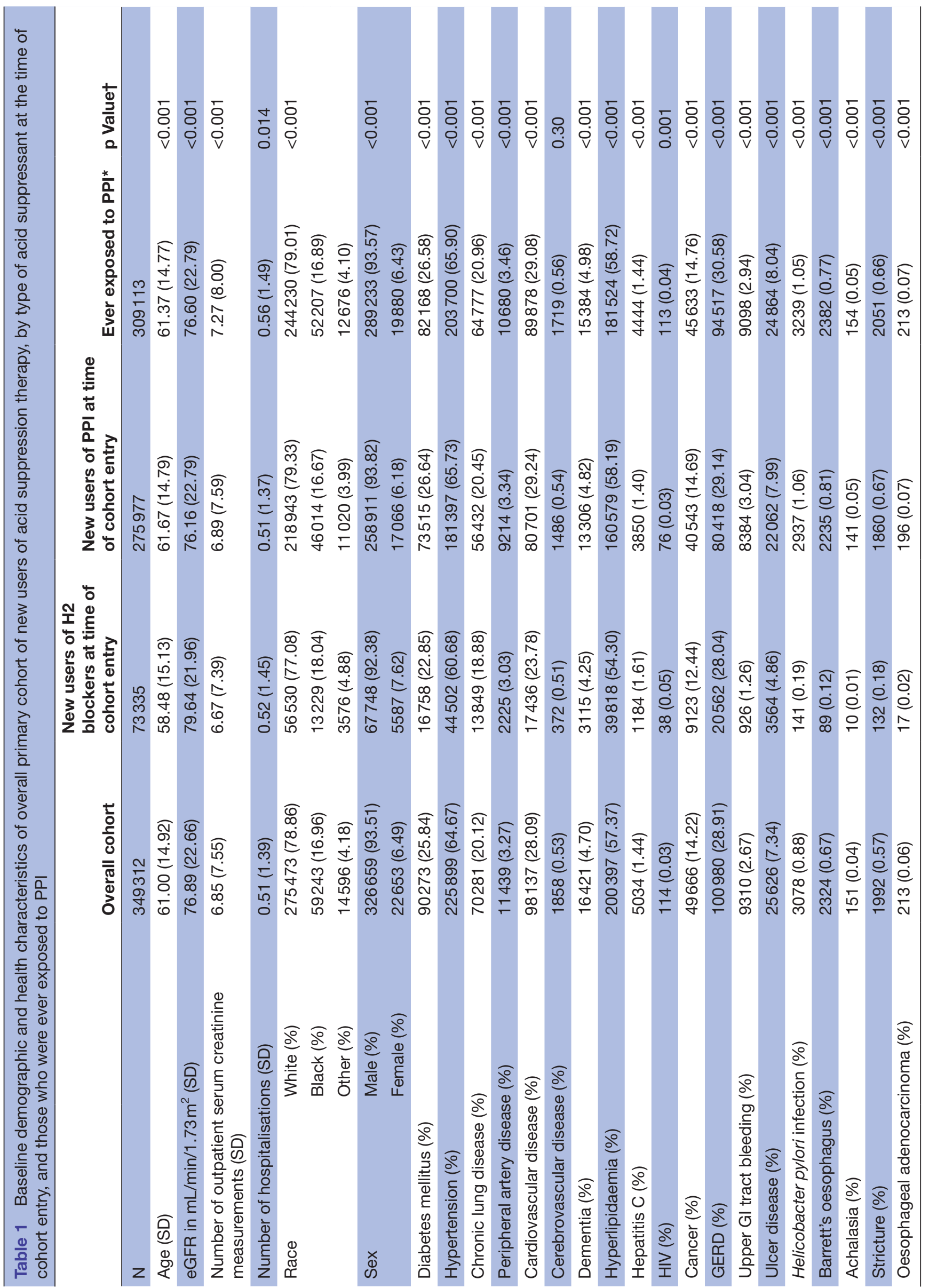




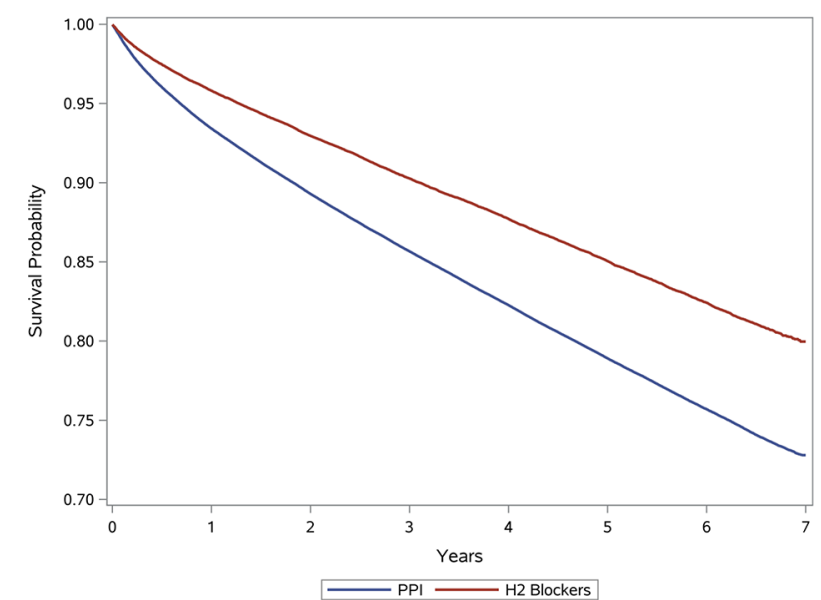

Figure 1 Survival curves for PPI and $\mathrm{H} 2$ blockers. PPI, proton pump inhibitor.

time dependent, PPI use was associated with increased risk of death compared with H2 blockers use (HR 1.17, CI 1.15 to 1.19$)$. In a 1:1 time-dependent propensity score-matched cohort of PPI and H2 blockers, PPI users had significantly increased risk of death HR 1.21 (CI 1.19 to 1.24). Furthermore, we also observed a graded association between cumulative duration of exposure to PPI and risk of death (see online supplementary table 2 and online supplementary figure 3 ).

To examine the potential impact of residual confounding on study results, we used rule-out and external adjustment approaches as described by Schneeweiss. ${ }^{41}$ Using the rule-out approach, we characterised a set of parameters (OR for relationship of PPI and confounder and HR for relationship of confounder and death) with sufficient strength to fully explain the association observed in primary analyses (see online supplementary figure 4). For example, if the confounder was two times as likely among PPI users $(\mathrm{OR}=2)$, and the HR of death associated with the uncontrolled confounder exceeded 4.0, then the uncontrolled confounder would fully explain the observed association between PPI and death (see online supplementary figure 4). Given that our analyses accounted for most known strong independent risk factors of death and employed an active comparator group, to cancel the results, any uncontrolled confounder of the required prevalence (OR 2 or more in the example above) and strength (HR 4 or more in the example above) would also have to be independent of the confounders already adjusted for and is unlikely to exist; thus, the results cannot be fully explained by this putative uncontrolled confounder.

External adjustment to estimate the impact of three unmeasured confounders, including obesity, smoking and use of therapeutics including anticoagulants, antiplatelet agents and non-steroidal anti-inflammatory drugs, shows a net confounding bias of $9.66 \%$ (see online supplementary figure 5 ). The total bias could move a null association between PPI and death from HR 1.00 to HR 1.10 (reflecting the net positive bias of $9.66 \%$ rounded up 
Table 2 Association between PPI use and risk of death

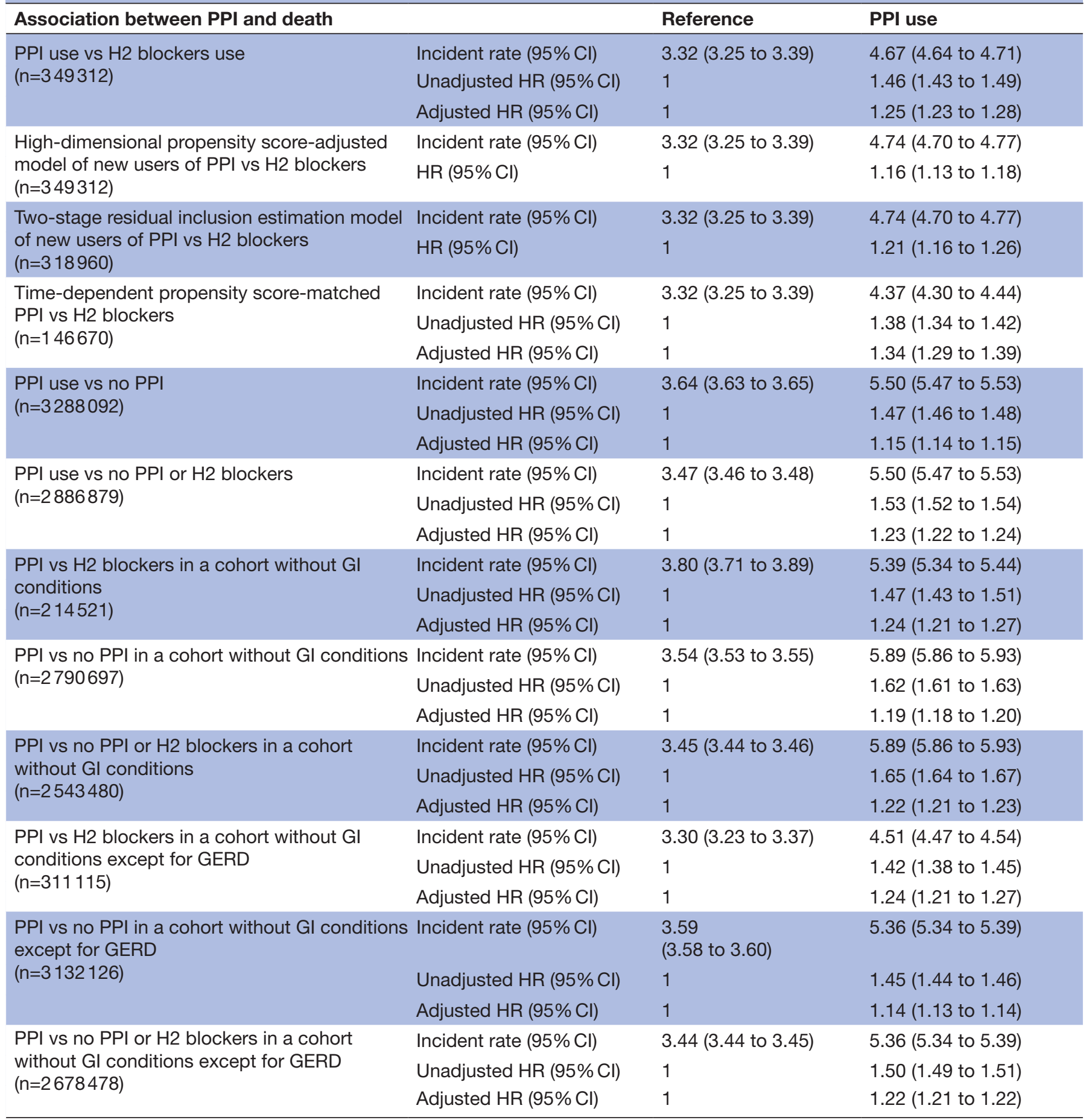

Incident rate as incident death in 100 person-years.

All models except time-dependent propensity score-matched and high-dimensional propensity score-adjusted models were time-dependent models. Effect of PPI was treated as time dependent and was defined as once patients used PPI, they were in PPI group during the remaining follow-up.

Adjusted model controlling for eGFR, age, race, gender, number of serum creatinine measurements, number of hospitalisations, diabetes mellitus, hypertension, cardiovascular disease, peripheral artery disease, cerebrovascular disease, chronic lung disease, hepatitis $\mathrm{C}$, HIV, dementia, cancer, GERD, upper GI tract bleeding, ulcer disease, H. pylori infection, Barrett's oesophagus, achalasia, stricture and oesophageal adenocarcinoma, unless used in analysis inclusion criteria.

Gl conditions include upper Gl tract bleeding, ulcer disease, $H$. pylori infection, Barrett's oesophagus, achalasia, stricture and oesophageal adenocarcinoma.

GERD, gastro-oesophageal reflux disease; GI, gastrointestinal; PPI, proton pump inhibitor. 
Table 3 Duration of exposure to PPI and risk of death among new users of PPI $(n=166098)$

\begin{tabular}{llllll}
\hline Duration (days) & $\mathbf{3 0}$ & $\mathbf{3 1 - 9 0}$ & $\mathbf{9 1 - 1 8 0}$ & $\mathbf{1 8 1 - 3 6 0}$ & $\mathbf{3 6 1 - 7 2 0}$ \\
\hline $\begin{array}{l}\mathrm{N} \\
(\%)\end{array}$ & $24748(14.90)$ & $39345(23.69)$ & $29334(17.66)$ & $33907(20.41)$ & $38764(23.34)$ \\
$\mathrm{HR}(95 \% \mathrm{Cl})$ & 1 & $1.05(1.02$ to 1.08$)$ & $1.17(1.13$ to 1.20$)$ & $1.31(1.27$ to 1.34$)$ & $1.51(1.47$ to 1.56$)$ \\
\hline
\end{tabular}

Within people exposed to PPI between 1 and 720 days.

Model controls for eGFR, age, race, gender, number of serum creatinine measurements, number of hospitalisations, diabetes mellitus, hypertension, cardiovascular disease, peripheral artery disease, cerebrovascular disease, chronic lung disease, hepatitis C, HIV, dementia, cancer, GERD, upper Gl tract bleeding, ulcer disease, Helicobacter pylori infection, Barrett's oesophagus, achalasia, stricture and oesophageal adenocarcinoma.

Time zero defined as date when the patient's last PPI prescription ends.

GERD, gastro-oesophageal reflux disease; GI, gastrointestinal; PPI, proton pump inhibitor.

to $10.0 \%$ ). The association we observed between PPI and death was $1.25>1.10$, which cannot be fully due to bias of unmeasured confounding.

In analyses where time-dependent exposure was classified as current use (within 90 days), past use (use prior to 90 days) and never use of PPI, compared with use of H2 blockers and never use of PPI (the reference group), current use of PPI and past use of PPI were associated with increased in risk of death (HR 1.23, CI 1.21 to 1.26, and HR 1.53, CI 1.50 to 1.57, respectively).

The association between PPI and death remained significant after excluding cohort participants who died within 90 days after the first PPI or H2 blocker prescription (HR 1.23, CI 1.20 to 1.26), or additionally controlling for haemoglobin levels (HR 1.25, CI 1.23 to 1.28). In models stratified for the presence of cardiovascular disease, history of pneumonia, CKD and age at T0, there was increased risk of death associated with PPI use in those with and without cardiovascular disease (HR 1.19, CI 1.15 to 1.23 , and HR 1.30 , CI 1.27 to 1.34 , respectively), with and without history of pneumonia (HR 1.39, CI 1.32 to 1.45, and HR 1.21, CI 1.18 to 1.24, respectively), with and without CKD (HR 1.18, CI 1.14 to 1.22, and HR 1.29, CI 1.26 to 1.33 , respectively) and above and below age 65 years (HR 1.17, CI 1.13 to 1.20, and HR 1.44, CI 1.39 to 1.50 , respectively). As a test of specificity, among users of acid suppression therapy, PPI use was not associated with increased risk of the tracer outcome of a motor vehicle accident (HR 0.99, CI 0.89 to 1.10).

\section{DISCUSSION}

This study provides insights into the excess risk of death associated with PPI use. In a large primary cohort of new users of acid suppression therapy followed for a median of 5.71 years, we show a significant association between PPI use and risk of all-cause mortality. Risk was increased among those with no documented medical indications for PPI use and with prolonged duration of use. The results were consistent in multiple analyses and robust to changes in epidemiological design and statistical specifications, and were reproduced in an earlier and less contemporary cohort from an era where PPI use was far less frequent. ${ }^{45}$
PPI are widely used by millions of people for indications and durations that were never tested or approved; they are available over the counter (without prescription) in several countries and generally perceived as safe class of therapeutics. They are often overprescribed, rarely deprescribed and frequently started inappropriately during a hospital stay, and their use extended for long-term duration without appropriate medical indication. ${ }^{46-50}$ Results of nationally representative data from the National Health and Nutrition Examination Survey, where analyses were weighted to represent the US adult population, showed that the use of prescription PPI increased from $3.9 \%$ to $7.8 \%$ from $1999-2000$ to 2011-2012, representing a doubling of prevalence ratio. ${ }^{45}$ Studies estimate that between $53 \%$ and $69 \%$ of PPI prescriptions are for inappropriate indications ${ }^{46}{ }^{51}$ where benefits of PPI use may not justify the risks for many users. ${ }^{51-53}$ The findings in our study highlight a potential excess risk of death among users of PPI, and in particular among cohort participants without GI comorbidities, and that risk is increased with prolonged duration of PPI exposure. Although our results should not deter prescription and use of PPI where medically indicated, they may be used to encourage and promote pharmacovigilance and emphasise the need to exercise judicious use of PPI and limit use and duration of therapy to instances where there is

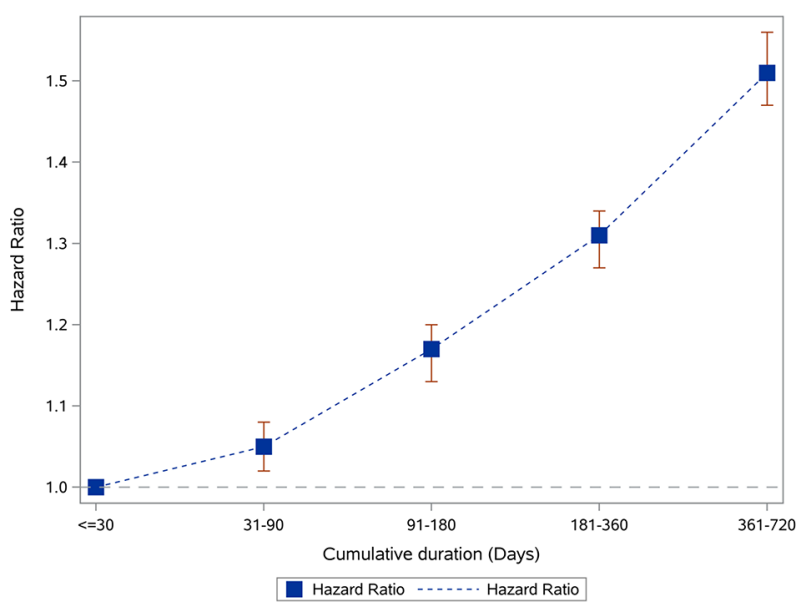

Figure 2 Duration of PPI exposure and risk of death among new PPI users ( $n=166098)$. PPI, proton pump inhibitor. 
a clear medical indication and where benefit outweighs potential risk. ${ }^{1}$ Standardised guidelines for initiating PPI prescription may lead to reduced overuse,${ }^{54}$ regular review of prescription and over-the-counter medications and deprescription where a medical indication for PPI treatment ceases to exist may be a meritorious approach. ${ }^{52}$

The biologic mechanism underpinning the association of PPI use and risk of death is not clear. Experimental evidence in rats suggests that PPI administration limits the regenerative capacity of livers following partial hepatectomy. ${ }^{55}$ Administration of PPI upregulates expression of mRNA and protein level and results in increased activity of the heme oxygenase- 1 enzyme in gastric and endothelial cells. ${ }^{56}$ Heme oxygenase-1 is generally seen as salutary, but its beneficial properties are vitiated at higher doses, and with sustained duration of expression. ${ }^{57}$ PPI treatment impairs lysosomal acidification and proteostasis and results in increased oxidative stress, dysfunction, telomere shortening and accelerated senescence of human endothelial cells. ${ }^{18} 58 \mathrm{Wu}$ and collaborators undertook a systematic toxicity mechanism analysis using a highthroughput in silico analysis of microarray data; they reported that PPI upregulated genes in the cellular retinol metabolism pathway and downregulated genes in the complement and coagulation cascades pathway, and that PPI may block pathways of antigen presentation and abrogate the synthesis and secretion of cytokines and complement component proteins and coagulation factors. ${ }^{5859}$ How the changes in gene expression contribute to excess risk of death is not yet entirely clear. The plausible clinical course leading to heightened risk of death is likely mediated by the occurrence of one or more of the adverse events associated with PPI use (kidney disease, dementia, hypomagnesemia, C. difficile infection, osteoporotic fracture and so on). Further studies are needed to characterise the biologic mechanisms that might explain the epidemiological findings in this report.

The constellation of findings in this report must be interpreted with the full cognizance of the observational study design where confounding by indication and selection bias may represent limitations. We employed an analytic strategy to evaluate the risk of death among users of acid suppression therapy (PPI and H2 blockers), a class of therapeutics generally prescribed for similar indications, a strategy that may lessen but does not completely eliminate the possibility of confounding by indication bias. We additionally built time-dependent propensity score-matched cohort and high-dimensional propensity score-adjusted models, and we employed the use of instrumental variable to reduce potential confounding bias. Although we accounted for known covariates in our analyses, it is possible that there are residual confounders (either unmeasured or unknown) that may still confound the association of PPI and risk of death. However, we evaluated the impact of residual confounding in quantitative bias analyses, and the results suggest that even with the application of unlikely (and exaggerated) set of assumptions, the risk cannot be fully explained by residual confounding. In our analyses, we defined drug exposure as having a prescription for it. Because PPIs (and H2 blockers) are available over the counter in the USA, it is possible that some patients in this cohort may have obtained and used PPI without prescription. However, owing to financial considerations, this is not highly likely, and if it occurred in some patients, it will have biased the results against the primary hypothesis and resulted in underestimation of risk. The cohort included mostly older white male US veterans, which may limit the generalisability of study results to a broader population. Our data sets did not include information on the cause of death. The study has a number of strengths, including the use of national large-scale data from a network of integrated health systems, which were captured during routine medical care that minimises selection bias. We employed a new user (incident user) approach and evaluated the association between PPI use and risk of death using a number of analytical approaches where we consistently found a significant association between PPI use and increased risk of death. The consistency of study findings in our report and the growing body of evidence in the literature showing a host of adverse events associated with PPI use are compelling, and because of the high prevalence of PPI use, it may have public health implications. Exercising pharmacovigilance and limiting PPI use to instances and durations where it is medically indicated may be warranted.

\section{Author affiliations}

${ }^{1}$ Clinical Epidemiology Center, Research and Education Service, VA Saint Louis Health Care System, Saint Louis, Missouri, USA

${ }^{2}$ Department of Medicine, Washington University School of Medicine, Saint Louis, Missouri, USA

${ }^{3}$ Department of Biostatistics, College for Public Health and Social Justice, Saint Louis University, Saint Louis, Missouri, USA

${ }^{4}$ Division of Public Health Sciences, Department of Surgery, Washington University School of Medicine, Saint Louis, Missouri, USA

${ }^{5}$ Renal Section, Medicine Service, VA Saint Louis Health Care System, Saint Louis, Missouri, USA

${ }^{6}$ Institute for Public Health, Washington University in Saint Louis, Saint Louis, Missouri, USA

Contributors Research area and study design: $Y X, B B, T L, H X, Y Y$ and $Z A A$; data acquisition: $Y X$ and $B B$; data analysis and interpretation: $Y X, B B, T L, H X, Y Y$ and ZAA; statistical analysis: YX and BB; supervision and mentorship: ZAA. Each author contributed important intellectual content during manuscript drafting or revision and accepts accountability for the overall work by ensuring that questions pertaining to the accuracy or integrity of any portion of the work are appropriately investigated and resolved. ZAA takes responsibility that this study has been reported honestly, accurately and transparently; that no important aspects of the study have been omitted; and that any discrepancies from the study as planned have been explained.

Disclaimer The contents do not represent the views of the US Department of Veterans Affairs or the US Government.

Competing interests None declared.

Ethics approval This research project was reviewed and approved by the Institutional Review Board of the VA Saint Louis Health Care System.

Provenance and peer review Not commissioned; externally peer reviewed.

Data sharing statement Data are available through the US Department of Veterans Affairs. 
Open Access This is an Open Access article distributed in accordance with the Creative Commons Attribution Non Commercial (CC BY-NC 4.0) license, which permits others to distribute, remix, adapt, build upon this work non-commercially, and license their derivative works on different terms, provided the original work is properly cited and the use is non-commercial. See: http://creativecommons.org/ licenses/by-nc/4.0/

(c) Article author(s) (or their employer(s) unless otherwise stated in the text of the article) 2017. All rights reserved. No commercial use is permitted unless otherwise expressly granted.

\section{REFERENCES}

1. Schoenfeld AJ, Grady D. Adverse effects associated with proton pump inhibitors. JAMA Intern Med 2016;176:172-4.

2. Xie Y, Bowe B, Li T, et al. Proton pump inhibitors and risk of incident CKD and progression to ESRD. J Am Soc Nephrol 2016;27:3153-63.

3. Antoniou T, Macdonald EM, Hollands S, et al. Proton pump inhibitors and the risk of acute kidney injury in older patients: a populationbased cohort study. CMAJ Open 2015;3:E166-E171.

4. Klepser DG, Collier DS, Cochran GL. Proton pump inhibitors and acute kidney injury: a nested case-control study. BMC Nephrol 2013;14:150.

5. Blank ML, Parkin L, Paul C, et al. A nationwide nested case-control study indicates an increased risk of acute interstitial nephritis with proton pump inhibitor use. Kidney Int 2014;86:837-44.

6. Lazarus B, Chen Y, Wilson FP, et al. Proton pump inhibitor use and the risk of chronic kidney disease. JAMA Intern Med 2016;176:238-46.

7. Xie Y, Bowe B, Li T, et al. Long-term kidney outcomes among users of proton pump inhibitors without intervening acute kidney injury. Kidney Int 2017;91:1482-94.

8. Gomm W, von Holt K, Thomé F, et al. Association of proton pump inhibitors with risk of dementia: a pharmacoepidemiological claims data analysis. JAMA Neurol 2016;73:410-6.

9. Danziger J, William JH, Scott DJ, et al. Proton-pump inhibitor use is associated with low serum magnesium concentrations. Kidney Int 2013;83:692-9.

10. Kieboom BC, Kiefte-de Jong JC, Eijgelsheim M, et al. Proton pump inhibitors and hypomagnesemia in the general population: a population-based cohort study. Am J Kidney Dis 2015;66:775-82.

11. Broeren MA, Geerdink EA, Vader HL, et al. Hypomagnesemia induced by several proton-pump inhibitors. Ann Intern Med 2009;151:755-6.

12. Kwok CS, Arthur AK, Anibueze Cl, et al. Risk of Clostridium difficile infection with acid suppressing drugs and antibiotics: meta-analysis. Am J Gastroenterol 2012;107:1011-9.

13. Zhou B, Huang $\mathrm{Y}$, Li H, et al. Proton-pump inhibitors and risk of fractures: an update meta-analysis. Osteoporos Int 2016;27:339-47.

14. Yu EW, Bauer SR, Bain PA, et al. Proton pump inhibitors and risk of fractures: a meta-analysis of 11 international studies. Am J Med 2011;124:519-26.

15. Melloni C, Washam JB, Jones WS, et al. Conflicting results between randomized trials and observational studies on the impact of proton pump inhibitors on cardiovascular events when coadministered with dual antiplatelet therapy: systematic review. Circ Cardiovasc Qual Outcomes 2015;8:47-55.

16. Filion KB, Chateau D, Targownik LE, et al. Proton pump inhibitors and the risk of hospitalisation for community-acquired pneumonia replicated cohort studies with meta-analysis. Gut 2014;63:552-8.

17. Eom CS, Jeon CY, Lim JW, et al. Use of acid-suppressive drugs and risk of pneumonia: a systematic review and meta-analysis. CMAJ 2011;183:310-9.

18. Yepuri G, Sukhovershin R, Nazari-Shafti TZ, et al. Proton pump inhibitors accelerate endothelial senescence. Circ Res 2016;118.

19. Bell JS, Strandberg TE, Teramura-Gronblad M, et al. Use of proton pump inhibitors and mortality among institutionalized older people. Arch Intern Med 2010;170:1604-5.

20. Maggio M, Corsonello A, Ceda GP, et al. Proton pump inhibitors and risk of 1-year mortality and rehospitalization in older patients discharged from acute care hospitals. JAMA Intern Med 2013;173:518-23.

21. Teramura-Grönblad M, Bell JS, Pöysti MM, et al. Risk of death associated with use of PPIs in three cohorts of institutionalized older people in Finland. J Am Med Dir Assoc 2012;13:488.e9-e13.

22. Wilson N, Gnjidic D, March L, et al. Use of PPIs are not associated with mortality in institutionalized older people. Arch Intern Med 2011;171:866.

23. Crowley $\mathrm{J}$. Covariance analysis of heart transplant survival data $\mathrm{Hu}$ Marie. J Am Stat Assoc 1977;72:27-36
24. Li T, Xie Y, Bowe B, et al. Serum phosphorus levels and risk of incident dementia. PLoS One 2017;12:e0171377.

25. Gawron AJ, Pandolfino JE, Miskevics S, et al. Proton pump inhibitor prescriptions and subsequent use in US veterans diagnosed with gastroesophageal reflux disease. J Gen Intern Med 2013;28:930-7.

26. Bowe $\mathrm{B}$, Xie $\mathrm{Y}, \mathrm{Xian} \mathrm{H}$, et al. Low levels of high-density lipoprotein cholesterol increase the risk of incident kidney disease and its progression. Kidney Int 2016;89:886-96.

27. Bowe $\mathrm{B}, \mathrm{Xie} \mathrm{Y}$, Xian $\mathrm{H}$, et al. High density lipoprotein cholesterol and the risk of all-cause mortality among U.S. veterans. Clin J Am Soc Nephrol 2016;11:1784-93.

28. Bowe B, Xie Y, Xian H, et al. Geographic Variation and US County Characteristics Associated With Rapid Kidney Function Decline. Kidney International Reports 2017;2:5-17.

29. Levey AS, Stevens LA, Schmid $\mathrm{CH}$, et al. A new equation to estimate glomerular filtration rate. Ann Intern Med 2009;150:604-12.

30. Xie Y, Bowe B, Xian H, et al. Estimated GFR Trajectories of People Entering CKD Stage 4 and Subsequent Kidney Disease Outcomes and Mortality. Am J Kidney Dis 2016;68:219-28.

31. Xie $\mathrm{Y}$, Bowe $\mathrm{B}$, Xian $\mathrm{H}$, et al. Rate of kidney function decline and risk of hospitalizations in stage 3A CKD. Clin J Am Soc Nephrol 2015;10:1946-55.

32. Xie Y, Bowe B, Xian H, et al. Estimated GFR trajectories of people entering CKD Stage 4 and subsequent kidney disease outcomes and mortality. Am J Kidney Dis 2016;68:219-28.

33. Xie $\mathrm{Y}$, Bowe $\mathrm{B}, \mathrm{Xian} \mathrm{H}$, et al. Renal function trajectories in patients with prior improved eGFR slopes and risk of death. PLoS One 2016;11:e0149283.

34. Schultz LR, Peterson EL, Breslau N. Graphing survival curve estimates for time-dependent covariates. Int J Methods Psychiatr Res 2002;11:68-74.

35. Lu B. Propensity score matching with time-dependent covariates. Biometrics 2005;61:721-8

36. Funk MJ, Westreich D, Wiesen $C$, et al. Doubly robust estimation of causal effects. Am J Epidemiol 2011;173:761-7.

37. Schneeweiss S, Rassen JA, Glynn RJ, et al. High-dimensional propensity score adjustment in studies of treatment effects using health care claims data. Epidemiology 2009;20:512-22.

38. Terza JV, Basu A, Rathouz PJ. Two-stage residual inclusion estimation: addressing endogeneity in health econometric modeling $\checkmark$ Health Econ 2008;27:531-43.

39. Adams AL, Black MH, Zhang JL, et al. Proton-pump inhibitor use and hip fractures in men: a population-based case-control study. Ann Epidemiol 2014;24:286-90.

40. Suissa S. Immortal time bias in observational studies of drug effects. Pharmacoepidemiol Drug Saf 2007;16:241-9.

41. Schneeweiss S. Sensitivity analysis and external adjustment for unmeasured confounders in epidemiologic database studies of therapeutics. Pharmacoepidemiol Drug Saf 2006;15:291-303.

42. GBD 2015 Risk Factors Collaborators. Global, regional, and national comparative risk assessment of 79 behavioural, environmental and occupational, and metabolic risks or clusters of risks, 1990-2015: a systematic analysis for the Global Burden of Disease Study 2015. Lancet 2016;388:1659-724.

43. GBD 2015 Mortality and Causes of Death Collaborators. Global, regional, and national life expectancy, all-cause mortality, and causespecific mortality for 249 causes of death, 1980-2015: a systematic analysis for the Global Burden of Disease Study 2015. Lancet 2016;388:1459-544.

44. Hvid-Jensen FNR, Pedersen L, Funch-Jensen P, et al. Thomsen RW Lifestyle factors among proton pump inhibitor users and nonusers: a cross-sectional study in a population-based setting. Dovepress 2013;5:493-9.

45. Kantor ED, Rehm CD, Haas JS, et al. Trends in prescription drug use among adults in the United States from 1999-2012. JAMA 2015;314:1818-31.

46. Forgacs I, Loganayagam A. Overprescribing proton pump inhibitors. BMJ 2008;336:2-3.

47. Choudhry MN, Soran H, Ziglam HM. Overuse and inappropriate prescribing of proton pump inhibitors in patients with Clostridium difficile-associated disease. QJM 2008;101:445-8.

48. Zink DA, Pohlman M, Barnes M, et al. Long-term use of acid suppression started inappropriately during hospitalization. Aliment Pharmacol Ther 2005;21:1203-9.

49. Strid H, Simren M, Bjornsson ES. Overuse of acid suppressant drugs in patients with chronic renal failure. Nephrology, dialysis, transplantation: official publication of the European Dialysis and Transplant Association-European Renal Association. Nephrol Dial Transplant 2003:18:570-5. 
50. Qato DM, Alexander GC, Conti RM, et al. Use of prescription and over-the-counter medications and dietary supplements among older adults in the United States. JAMA 2008;300:2867-78.

51. Katz MH. Failing the acid test: benefits of proton pump inhibitors may not justify the risks for many users. Arch Intern Med 2010;170:747-8.

52. Linsky A, Simon SR. Reversing gears: discontinuing medication therapy to prevent adverse events. JAMA Intern Med 2013;173:524-5.

53. Grady D, Redberg RF. Less is more: how less health care can result in better health. Arch Intern Med 2010;170:749-50.

54. Yachimski PS, Farrell EA, Hunt DP, et al. Proton pump inhibitors for prophylaxis of nosocomial upper gastrointestinal tract bleeding: effect of standardized guidelines on prescribing practice. Arch Intern Med 2010;170:779-83.
55. Kucuk HF, Akyol H, Kaptanoglu L, et al. Effect of proton pump inhibitors on hepatic regeneration. Eur Surg Res 2006;38:322-8.

56. Becker JC, Grosser N, Waltke C, et al. Beyond gastric acid reduction: proton pump inhibitors induce heme oxygenase-1 in gastric and endothelial cells. Biochem Biophys Res Commun 2006;345:1014-21.

57. Nath KA. Heme oxygenase-1 and acute kidney injury. Curr Opin Nephrol Hypertens 2014;23:17-24.

58. Wu D, Qiu T, Zhang Q, et al. Systematic toxicity mechanism analysis of proton pump inhibitors: an in Silico Study. Chem Res Toxicol 2015;28:419-30.

59. Liu W, Baker SS, Trinidad J, et al. Inhibition of lysosomal enzyme activities by proton pump inhibitors. $J$ Gastroenterol 2013;48:1343-52. 